\title{
Patterns of Adverse Transfusion Reactions in a Tertiary Care Centre of Rural Bengaluru, South India: A Step Towards Hemovigilance
}

\author{
Indrani Krishnappa*, Uma Bai K R and Raja Parthiban \\ Dept of Pathology, MVJ Medical College \& Research Hospital, Bangalore, INDIA
}

\begin{abstract}
Background: "Blood transfusion is like marriage: it should not be entered upon lightly, unadvisedly or wantonly or more often than is absolutely necessary." This statement from Robert Beal clearly indicates the necessity and potential risk of blood transfusion and thus the importance of "Hemovigilance".

Aims \& Objectives: The study was done to collect and analyse the frequency and nature of the transfusion reactions reported at a tertiary care hospital in Rural Bengaluru, Karnataka.

Materials \& Methods: A retrospective review of all transfusion reactions reported to the blood bank at the Transfusion Medicine department between April 2012 and December 2017 was done. All transfusion reactions were evaluated by the blood centre and classified under standard definitions.

Results: During the study period, 10114 units were transfused. Out of these $72(0.71 \%)$ cases of post transfusion reactions were reported. Most of our patients reported with transfusion reactions were young adults in the age group of 21-30 years. The most commonly encountered type of reaction was Febrile Non Hemolytic transfusion reaction (FNHTR) in 28 cases. Grade III reactions were reported in 5 cases, Transfusion associated Cardiac Overload (TACO) ( 3 cases) \& anaphylaxis ( 2 cases).

Conclusion: Transfusion reactions encountered in our study varied from innocuous ones like FNHTR to life threatening anaphylactic reactions. This underlines the need for constant monitoring and strict implementation of standard protocols for transfusion of blood and blood products. Our study also highlights the need for pre transfusion counselling and assurance to reduce anxiety among individuals undergoing transfusion.
\end{abstract}

Keywords: Blood Transfusion, Transfusion Related Reactions, Hemovigilance

\section{Introduction}

In 1901, Karl Landstiener discovered the ABO blood group system and laid foundation for the modern blood transfusion services. ${ }^{(1)}$ During the past few decades, transfusion practices have undergone a significant change. Emphasis is being laid on use of blood components instead of whole blood to reduce the adverse reactions associated with transfusion. The incidence of transfusion transmitted diseases (TTDs) has reduced drastically due to the availability of facilities for testing TTDs. However, the incidence of adverse events due to human error, alloimmunisation, bacterial contamination and immunomodulation phenomenon remain a matter of concern. ${ }^{(2)}$

Hemovigilance is defined as a set of surveillance procedures covering whole transfusion chain from the collection of blood and its components to the follow up of its recipients, intended to collect and access information on unexpected or undesirable effects resulting from the therapeutic use of labile blood products, and to prevent their occurrence and recurrence. ${ }^{(3)}$ In Europe, the first hemovigilance programme began in France in 1994 \& in the United Kingdom in 1996. Reporting to the recent US biovigilance network started as recently as 2008 only. (4) Well functioning hemovigilance systems indicate how safety can be improved and also document the success of various measures taken towards safe transfusion practices. ${ }^{(5)}$ Reporting of a transfusion reaction is essential for patient care \& management. However there is underreporting of most of the minor transfusion reactions by the medical staff, therefore the exact incidence of transfusion reactions is not known. In our study we have evaluated the various transfusion reactions as a pilot institutional effort towards an effective hemovigilance programme.

\section{Materials and Methods}

We performed a retrospective observational analysis of data available in our blood bank records of transfusion related reactions for a period of five years and eight months from April 2012 to December 2017. Cases of transfusion reaction occurring in our hospital and reported to the blood bank immediately where all the clinical details and transfusion reaction work up particulars were available were included in the study. 
After the issue of blood unit from the blood bank, within half an hour of issue transfusion was started in the presence of a resident/ nurse. When a reaction occurred, the concerned physician/resident performed a preliminary analysis and reported the same to the blood bank. A standard "transfusion reaction proforma/form" was used comprising of the following details of the patient: patient identification details, clinical diagnosis, indication for transfusion, component of blood transfused, number of units transfused, time of issue of blood unit, time of commencement of transfusion, time of completion of transfusion, time of occurrence of transfusion reaction , signs and symptoms of reaction like fever, chills/rigor, pruritus, skin rash, urticaria, respiratory distress, nausea/ vomiting, headache, chest/ flank/back pain, hypotension, hypertension, tachycardia, vein pain, disturbance of consciousness and hemoglobinuria. The proforma also included the post transfusion work up of the patients.

Post transfusion work up included examination of the whole blood or blood component unit returned to the blood bank if transfusion had to be stopped during transfusion, direct and indirect Comb's test on fresh patient blood samples, urine examination for signs of hematuria and a repeat blood grouping \&cross matching of the patient and donor samples.

All transfusion reactions were evaluated by the blood centre and classified under standard definitions provided by International Society of Blood Transfusion(ISBT). The reactions were classified into immune and non immune reactions. Immune reactions included Febrile Non Haemolytic Transfusion Reaction (FNHTR), allergic reaction, anaphylaxis and alloimmunization. Non immune reaction included Transfusion Associated Cardiac Overload (TACO). The reactions were also graded into four grades based on the ISBT guidelines. The frequency of transfusion reactions was calculated for each type of component transfused. Statistical analysis was performed using Microsoft excel software.

\section{Results}

Number of Transfusions: During this study, 10114 units were transfused. Out of this there were 3802 units of pRBCs, 2813 units of whole blood, 2067 units of platelet concentrates and 1432 units of Fresh Frozen Plasma (FFP). The number of pRBCs administered represented $37.6 \%$ of total transfused units. The number of whole blood, platelet concentrate and FFPs represented 27.8, 20.4 and 14.15 per cent respectively (Figure 1).

Transfusion Reactions following Transfusion of blood components: In our study we encountered, $72(0.71 \%$ of total number of transfusions) cases of transfusion related reactions. Most of the cases were seen in adults in the age group of 21 to 30 years. The oldest patient in our study was an 88 year old female transfused with whole blood and presented with severe dyspnoea post transfusion, was diagnosed as Transfusion Associated Cardiac Overload (TACO). The youngest patient in our study was an eight year old female who presented with fever and chills post transfusion of $300 \mathrm{ml}$ of whole blood. The various types of transfusion reactions encountered are shown in figure 2.

On a per transfused unit basis, $46(1.2 \%)$ of 3802 units of RBCs, 23 (0.81\%) of 2813 units of whole blood, $2(0.09 \%)$ of 2067 units of platelet concentrates and $1(0.69 \%)$ of 1432 units of FFP administered were associated with a transfusion reaction (Figure 3). Therefore the maximum rates of transfusion reactions were associated with transfusion of pRBCs followed by whole blood. Rarely reactions occurred following platelet and FFP transfusion.

Characteristics of Clinical Signs and Symptoms associated with Transfusion Reactions:

During the period of study, 5 cases of "life threatening grade III" transfusion reactions occurred. Anaphlactoid reactions were seen in 3 cases, transfused with one unit of $\mathrm{pRBC}$ each. Acute respiratory distress was seen in 2 of the patients during transfusion of whole blood unit, these were diagnosed as Transfusion Associated Cardiac Overload (TACO). Rest of the cases were graded as grade I (56 cases) and grade II (11 cases) based on the grading system recommended by ISBT. No cases of "Death - grade IV" reaction were seen in our study.

We evaluated all the reactions for their strength of relation to the transfusion of blood or blood components. We found that eleven cases which were reported to our blood bank as transfusion reactions were actually unrelated to transfusion and were confirmed during post transfusion work up of the patients. Six of these patients presented only with anxiety and post transfusion work showed no evidence of hemolysis in blood or urine. Nausea and vomiting were the complaints in two of the cases, these patients were diagnosed to have gastritis on admission itself and also post transfusion work up was negative. One of the patients presented with hematuria post transfusion, however this patient had undergone percutaneous nephrolithotomy and the hematuria was secondary to the procedure and not transfusion per se. The other patient presented to the Medicine OPD with fever and chills and was diagnosed to be suffering from urinary tract infection, these symptoms persisted post transfusion as well and the clinician falsely reported it as a transfusion reaction. None of these cases showed positive results on post transfusion work up of blood or urine. Transfusion was the cause of reactions "definitely" in 3 cases, "probably" in 33 cases and "possibly" in rest of the 36 cases. 
Table 1: Incidences of transfusion reactions (TRs) in various studies.

\begin{tabular}{|c|c|c|}
\hline Study & Rate of TRs & Most common type of TR \\
\hline Venkatachalapathy TS $^{(2)}$ & $3.3 \%$ & Allergic reactions \\
\hline Kumar P et al $^{(6)}$ & $0.18 \%$ & FNHTR \\
\hline${\text { Bhattacharya et } \mathbf{l}^{(7)}}^{(7)}$ & $0.26 \%$ & FNHTR \\
\hline Lubart et al $^{(8)}$ & $10.5 \%$ & FNHTR \\
\hline Pedrosa AKKV et al $^{(9)}$ & $3.8 \%$ & Allergic reactions \\
\hline Sharma DK et al ${ }^{(10)}$ & $0.92 \%$ & Allergic reactions \\
\hline Vartak UC et al $^{(11)}$ & $0.16 \%$ & FNHTR \\
\hline Bassi $R$ et al ${ }^{(12)}$ & $0.40 \%$ & FNHTR \\
\hline Present study & $0.71 \%$ & FNHTR \\
\hline
\end{tabular}

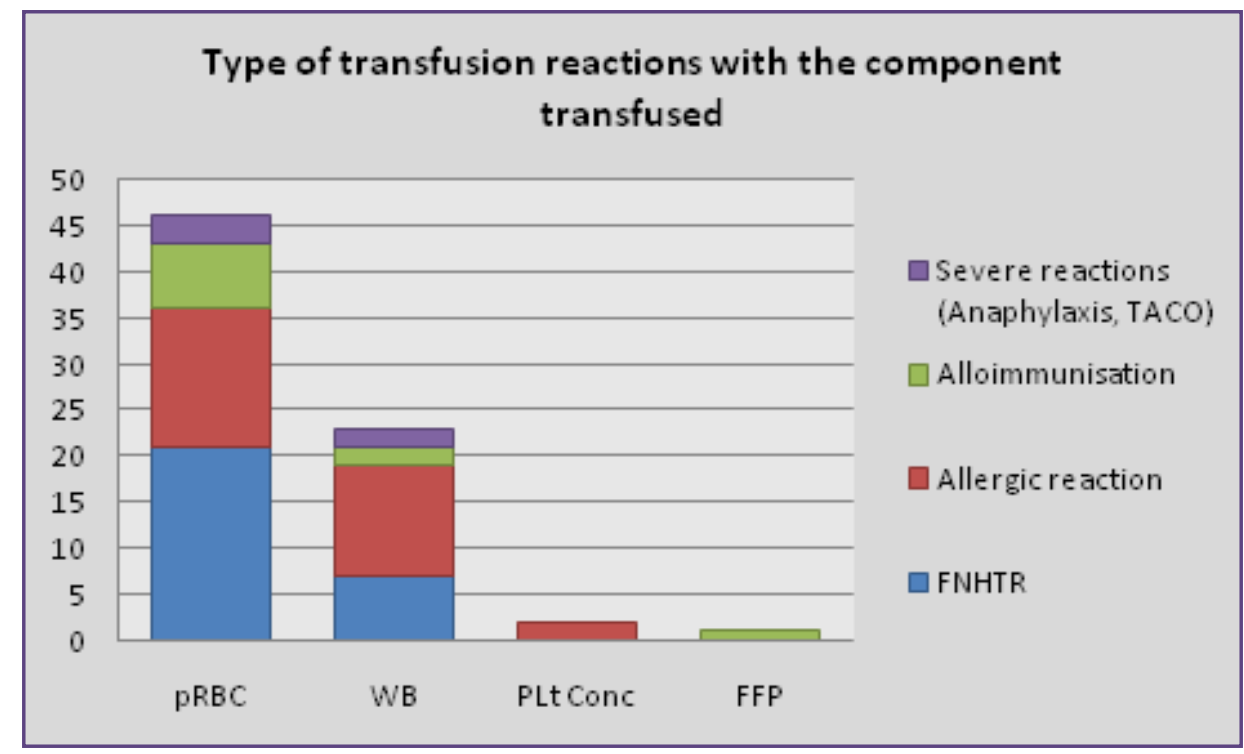

Fig. 1: Details of transfusions during the study period.

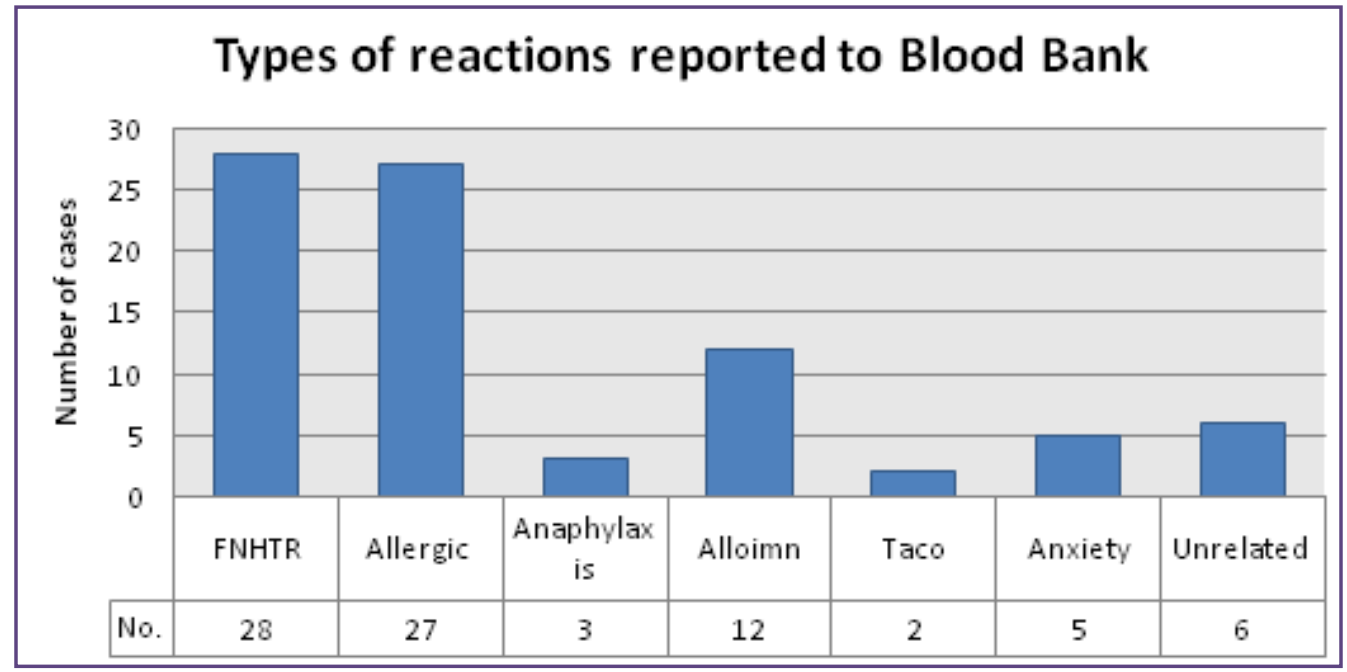

Fig. 2: Types of transfusion reactions (FNHTR - Febrile non haemolytic transfusion reaction, TACO - Transfusion Associated Cardiac Overload, Alloimn - Alloimmunisation, Unrelated - unrelated to transfusion). 


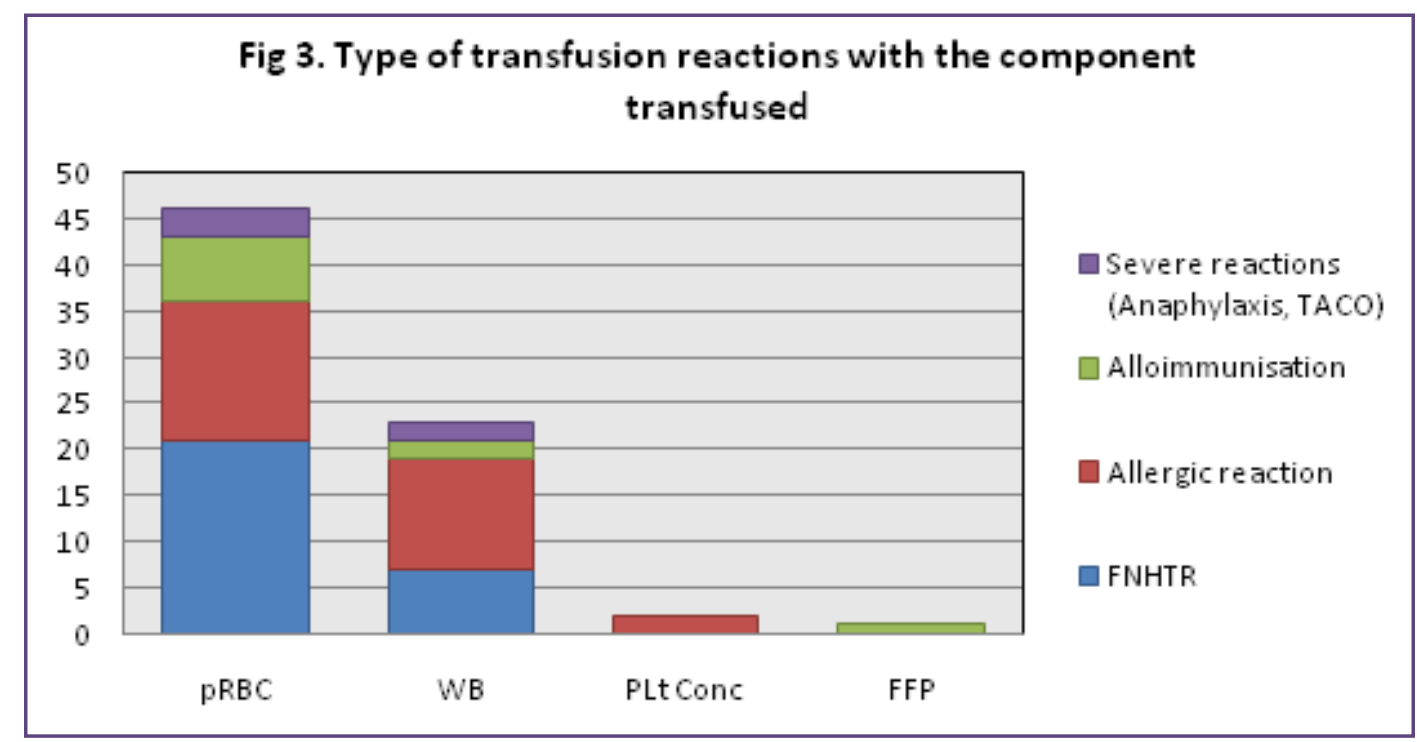

Fig. 3: Type of transfusion reactions with the component transfused (pRBC- Packed RBCs, WB - Whole blood, PltConc Platelet concentrate, FFP - Fresh Frozen Plasma).

\section{Discussion}

Adverse transfusion reactions are unprecedented risks that are associated with allogenic blood transfusions. Transfusion reactions present as adverse signs and symptoms occurring in patients during or after transfusion of blood components. These can be immediate or delayed. They might be associated with hemolysis or may be non hemolytic.

The frequency of transfusion reactions in our study was $0.71 \%$. This is much less, compared to the frequency of transfusion reactions noted in a study conducted by Venkatachalapathy TS at Government general hospital at Puducherry $(3.30 \%) .{ }^{(2)}$ This might be due to underreporting of milder transfusion related adverse reactions in our hospital. Therefore arises the need for strict implementation and training of consultants and nursing staff about Hemovigilance. However our results pertaining to the rate of transfusion reactions were comparable to findings of other authors. (Table 1)

Lubart et al had high incidence of transfusion reactions in their study because their study population was hospitalized elderly individuals who are frail with a high rate of comorbidities. ${ }^{(8)}$ The rate of transfusion reaction was higher $(3.8 \%)$ in the study conducted by Pedrosa AKKV et al as their study included only paediatric population. The prevalence of transfusion reactions in children is high, and the intervening factors are: type of blood component, age, patient comorbidity, and multiple transfusions and type of blood component transfused. ${ }^{(9)}$ The instances of transfusion reactions are higher in such population; unlike in our study where the predominant population were young adults in the age group of 21-30 years.

Maximum number of transfusion reactions in our study followed transfusion of pRBCs $(63.8 \%)$. Kumar $\mathrm{P}$ et al in their study found $42.8 \%$ of the reactions following pRBC transfusion. ${ }^{(6)}$ Hod EA et al have hypothesised that a subset of damaged RBCs from prolonged storage deliver large amounts of nontransferrin bound iron (NTBI) to the monocyte/macrophage system inducing inflammation. The increased NTBI also enhances bacterial growth in vitro which might be the cause of reactions occurring post $\mathrm{pRBC}$ transfusion. ${ }^{(13,14)}$ Therefore the incidence of transfusion related reactions post $\mathrm{pRBC}$ transfusion can be reduced by issuing relatively fresh units (that is in terms of fewer days of in house storage) to patients who are predisposed to such reactions.

Most of the reactions observed in our study were FNHTR (34\%), followed by allergic reactions (33\%).FNHTR are non haemolytic transfusion reactions caused by proinflammatory cytokines such as interleukin (IL) 1 beta, IL-6 and tumour necrosis factor (TNF) alpha released from WBCs and accumulated in blood components during storage or antibodies present in the donor blood/ blood component. ${ }^{(15)}$ The incidence of FNHTR was similar to that quoted by Kumar P et al (35.7\%)in their study. ${ }^{(6)}$ Heddle et al reported plasma depletion to be more effective than poststorage leukocyte reduction in preventing transfusion reactions such as FNHTRs and allergic reactions. ${ }^{(15)}$

Most of the allergic reactions in our study occurred post transfusion of whole blood (12 cases). The definitions for 
allergic reaction have varied from presence of only hives or urticaria, to presence of wheezing and angioedema as well in some studies. ${ }^{(16)}$ The reason for the high rate of allergic reactions following whole blood \&platelet concentrate transfusion is because the amount of plasma is more in these units. ${ }^{(17)}$ Therefore the incidence of allergic reactions can be brought down by plasma reduction and administering antihistaminics to the recipient prior to transfusion.

Alloimmunisation is immune response against donor antigens post transfusion of whole blood or blood components, generally occurring after multiple blood transfusions or pregnancies. Pretransfusion blood grouping, compatibilization and screening for the most immunogenic antibodies is normally performed prior to transfusion. However there are numerous other alloantibodies which are not tested for, as the titre of these antibodies might be very low and thus are not detectable. ${ }^{(18,19)}$ These can give rise to transfusion related reactions, mostly post transfusion of whole blood, packed cells or platelet concentrates. In our study, we came across twelve cases of alloimmunisation. A positive direct Comb's test was seen in three of these cases. We could not test for minor alloantibodies in these patients and hence the diagnosis was made based on clinical details and symptoms.

The ISBT "Working party on Hemovigilance" in 2011 have proposed standard definitions for surveillance of non infectious adverse transfusion reactions and graded the reactions based on their severity into non severe (grade I), severe (grade II), life threatening (grade III) and death following transfusion (grade IV). In our study most of the reactions (56 cases) were non severe (grade I), 11 cases were severe (grade II) and 5 cases were life threatening. Anaphlactoid reactions occurred in 3 cases following transfusion of pRBCs. Anaphylaxis is described as a severe allergic reaction when in addition to mucocutaneous symptoms there is airway compromise or severe hypotension. ${ }^{(20)}$ Hypotension can occur singly or as a part of other transfusion reactions like acute haemolytic reaction, bacterial contamination, transfusion-related acute lung injury or anaphylaxis. ${ }^{(11)}$ Hypotensive reactions occur due to activation of intrinsic contact activation pathway of coagulation cascade, generating bradykinin. ${ }^{(21)}$ In our cases of anaphylactic reaction, the patients manifested with dyspnoea and hypotension during transfusion. Hypotensive reactions tend to occur in patients taking antihypertensives, therefore one has to be cautious before transfusing such patients.

Transfusion associated cardiac overload (TACO) was reported in 2 cases in our study. TACO is characterised by acute respiratory distress, tachycardia, increased blood pressure, acute onset pulmonary edema or evidence of positive fluid balance within 6 hours of transfusion, according to NHSN. These criteria are similar to those mentioned by UK serious hazards of transfusion (SHOT) National Hemovigilance scheme with a shorter timeline (4 hrs) for development of these signs \&symptoms. ${ }^{(21)}$

Rarely reactions reported to the blood bank post transfusion do not account to adverse transfusion reactions and the cause lies in the medical condition of the patient. The ISBT "Working committee on Hemovigilance" has proposed various categories to assess the strength of relation to the transfusion of the adverse event. The categories are "definite, probable, possible, unlikely and excluded".(20) After the initial assessment of the adverse reaction, only reactions categorised as definite, probable or possible shall be considered as "transfusion related reactions" for international comparisons. In our study, we had 11 cases of 83 cases, where after the post transfusion investigations and clinical examination the reactions were categorised under "excluded" (6 cases) or "unlikely" (5 cases of anxiety) that is the events were unrelated to transfusion. Rest of the cases were categorises under "definite" (3 cases), "probable" (33 cases) and "possible" (36 cases) categories. Hence it is necessary to communicate and educate the nursing staff and residents regarding the reporting of transfusion related reactions, so that there can be efficient management of the patient during and post transfusion.

\section{Conclusion}

To summarise, transfusion of blood and blood components is always associated with a potential risk. This risk varies based on patient factors like age, blood component transfused and duration of in house storage of the unit transfused. Most commonly observed reactions in our study were FNHTR, which were managed conservatively. We also encountered three cases of life threatening anaphylactic reaction. Therefore we should always be vigilant and anticipate such reactions especially in the elderly individuals and patients with other co morbidities like compromised cardiac function. We also reported eleven cases of reactions unrelated to transfusion, majority being "anxiety". This highlights the need for pre transfusion counselling and assurance to the patients regarding the pros and cons of transfusion.

The national hemovigilance programme, at national level is working on documenting the true incidence $\&$ spectrum of transfusion reactions, so that policies can be formulated to minimize risk of adverse reactions post transfusion. Our study is an initial effort towards the National Hemovigilance 
programme. In future more elaborate analyses have to be performed to improve transfusion practices and devise methods to minimize these transfusion related adverse events.

\section{References}

1. Moore SB, Taswell HF, Pinedaa AA, Sonnenberg CL. Delayed haemolytic transfusion reactions: Evidence of the need for an improved pretransfusion compatibility test. Am J Clin Path.1980;74: 94-97.

2. Venkatachalapathy TS. A prospective audit of blood transfusion reactions in tertiary care hospital for the use of blood and blood components. J Blood DisordTransfus. 2012;3:118

3. Jain A, Kaur R. Hemovigilance and blood safety. Asian J Transfus Sci. 2012 Jul-Dec; 6(2): 137-138.

4. Kato H, Uruma M, Okuyama Y, Fujita H, Handa M, Tomiyama $\mathrm{Y}$ et al.Incidence of transfusion-related adverse reactions per patient reflects the potential risk of transfusion therapy in Japan. Am J ClinPathol2013;140:219-224.

5. De Vries RR, Faber JC, Strengers PF. Hemovigilance: An effective tool for improving transfusion practice. Vox Sang.2011;100:60-7.

6. Kumar P, Thapliyal R, Coshic P, Chatterjee K. Retrospective evaluation of adverse transfusion reactions following blood product transfusion from a tertiary care hospital: a preliminary step towards hemovigilance. Asian J TransfusSci. 2013;7(2):109-115.

7. Bhattacharya P, Marwaha N, Dhawan HK, Roy P, Sharma RR. Transfusion-related adverse events at the tertiary care center in North India: an institutional hemovigilance effort. Asian J TransfusSci. 2011; 5(2):164-170.

8. Lubart E, Segal R, Tryhub N, Sigler E, Leibovitz A. Blood transfusion reactions in elderly patients hospitalized in a multilevel geriatric hospital. J of Aging Research 2014:1-3.

9. Pedrosa AK, Pinto FJ, Lins LD, Deus GM. Blood transfusion reactions in children: Associated factors. J Pediatr.2013;89(4):400-6.

10. Sharma DK, Datta S, Amlan G. Study of acute transfusion reactions in a teaching hospital of Sikkim: A hemovigilance initiative. Indian J Pharmacol. 2015; 47(4): 370-374.
11. Vartak UC, Shewale R, Vartak S, Faizal F, Majethia N. Adverse reactions of blood transfusion: a study in a tertiary care hospital. International journal of scientific study.2016;4(2): 90-95.

12. Bassi R, Aggarwal S, Bharadwaj K, Thakur KK. Patterns of adverse transfusion reactions in a tertiary care centre of North india: a step towards hemovigilance. Int J Hematology and Blood transfusion. 2017;33(2):248-253.

13. Hod EA, Zhang N, Sokol SA, Francis RO, Ansaldi D, Francis $\mathrm{KP}$ et al. Transfusion of red blood cells after prolonged storage produces harmful effects that are mediated by iron and inflammation. Blood. 2010;115(21):4284-4295.

14. Goodell PP, Uhl N, Mohammed M, Powers AA. Risk of Hemolytic Transfusion Reactions Following EmergencyRelease RBC Transfusion.Am J ClinPathol. 2010;134:202206.

15. Heddle NM. Pathophysiology of febrile nonhemolytictransfusion reactions.Curr OpinHematol. 1999;6:420-426.

16. Sinha RTK, Rai P, Dey A. A retrospective evaluation of adverse transfusion reactions at a tertiary care centre in central India. Journal of Medical Sciences and Health. 2016;2(3):6-12.

17. Heddle NM, Blajchman MA, Meyer RM, et al. A randomized controlled trial comparing the frequency of acute reactions to plasma removed platelets and prestorage WBC-reduced platelets. Transfusion 2002; 42: 556-66.

18. Alves VM, Martins PRJ, Soares S, Araújo G, Schmidt LC, Costa SSM. Alloimmunization screening after transfusion of red blood cells in a prospective study. Rev Bras HematolHemoter. 2012;34(3):206-11.

19. Dlimi BH, Achenbach S, Strobel J, Eckstein R, Zimmermann R. Prevention and management of transfusion-induced alloimmunization: current perspectives. InternationalJournal of Clinical Transfusion Medicine 2014:2 59-63.

20. Proposed standard definitions for surveillance of non infectious adverse transfusion reactions: ISBT Working Party on Hemovigilance, Cape Town 2011.

21. Delaney M, Wendel S, Bercovitz RS, Cid J, Cohn C, Dunbar NM. Transfusion reactions: prevention, diagnosis, and treatment. The Lancet 2016;388:2825-2836.

*Corresponding author:

Dr. Indrani Krishnappa, Assistant Professor, Dept of Pathology, MVJMC \& RH, Dandupalya, Kolathur post, Hoskote, Bangalore, INDIA

Phone: +91 9740146525

Email: indranipramodh@gmail.com

Financial or other Competing Interests: None. 